\title{
DENTAL PATHOLOGY IN PREGNANCY
}

\author{
By Seymour Robinson, F.D.S. R.C.S.(Eng.) \\ Consulting Dental Surgeon, Westminster Children's Hospital, London
}

The correlation of diseases of the teeth with the reproductive system is first found in the writings of Hippocrates (460 B.C.). Throughout the ages writers have confirmed this relation. It has been appreciated for many years that a haematogenous infection of the reproductive organs may arise from a mouth or throat infection, whilst conversely, by lowering the body defences, pregnancy may predispose to dental infections.

In 1935 Okell and Elliott demonstrated that organisms may find their way into the blood stream when teeth are extracted. Later, Round, Kirkpatrick and Hails (1936) showed that even the chewing of food may produce a transient bacteriaemia in cases of pyorrhoea. The streptococci found in the blood stream were mostly of the streptococcus viridans type.

Fish and Maclean (1936) have explained that in cases of chronic gingivitis a layer of leucocytes prevents the organisms from entering the tissues so long as the ulcers at the gum margin are at rest. Any disturbance of the lesions tears the ulcer open and allows the organisms into the tissues or into the blood stream. Although in the majority of cases the organisms are destroyed there is the danger of their becoming localized and a recurring bacterial shower of haemolytic streptococci may produce anaemia by actual haemolysis although the organisms are destroyed.

During parturition it is possible that chance organisms may be shed into the uterus and may remain there for a time in a blood clot. Correct parodontal treatment undertaken during pregnancy should aim at the elimination of the gingival ' pockets,' thus removing the source of bacteria in the mouth. Such treatment is never contraindicated except perhaps in cases requiring extensive gingivectomy between the third and seventh months. The patient's medical attendant will readily comprehend the comparative risks between the minor operation of gingivectomy and the result of neglect and delay.

\section{The Gums}

It is well known that during pregnancy the gums, like other keratinized mucous membranes of the body, soften and are easily torn. It has been shown histologically (Ziskin et al., 1933) that oedema of the buccal epithelium occurs together with a thinning of the keratinized cuticle. It is important, therefore, that care be paid to the gum margins and an adequate degree of keratinization maintained. With healthy gums the mouth will be clean and the problem of taking care of the teeth is much simplified.

Clinically the gingivae during pregnancy may present various morbid states that are not necessarily caused by the same factors. In the first place the gums bleed easily when traumatized by the tooth brush, and the tendency to bleed is increased if the gums are not in good condition prior to the onset of the pregnancy. Secondly, the margin of the gums becomes inflamed and is of $a_{\infty}$ dull red colour. It bleeds profusely when probed and has been called the ' raspberry red gum' (Miller, 1946). The front of the mouth is more usually affected. In the next stage there is a generalized hypertrophy of the mouth tissues and the gums are swollen and often bright red. The gum papillae become hypertrophied and may grow to cover a large surface of the tooth itself. The gums bleed easily but are seldom painful, the condition being known as the "hypertrophic gingivitis of pregnancy,' although it is more a cellular infiltration and inflammatory hyperplasia than a true hypertrophy.

In another type large proliferations form in localized areas and protruding masses of hyperplastic tissue, either sessile or pedunculated, push out from the papillae. This form was described by Blum (I93I) as the 'pregnancy tumour.' When large it resembles a fibrous epulis and trauma from mastication produces an area of greyish necrosis on its surface. It differs from an epulis in that it will either disappear or diminish considerably after parturition.

A hypertrophic gingivitis in a non-pregnant woman may be continued during pregnancy, but the majority of gingival changes occur at times of hormonal activity, i.e. during pregnancy, menstruation and puberty. Ziskin (1937) and others have published details of experiments pointing to hormonal disturbances as aetiological factors in the pregnancy type of gingivitis. 


\section{Parodontal Disease}

This is a chronic infective process of the gingivae and adjacent tissues, and is characterized by a progressive destruction of the supporting structures of the teeth. The gum margins recede and in the final stage the teeth are extruded. A free discharge of pus is always found in the later stages.

Cause. The disease is common in all civilized races and at the outset is due to a lack of hardness of the keratinized layer of the gum epithelium. Cooked foods and the use of knives and forks are perhaps the main cause of 'soft' gums. The high crown of civilized man's tooth due to lack of grinding results in the softening of the gum epithelium for want of roughage and massage during mastication. The gum papillae become torn and mouth bacteria are pushed into the subcutaneous tissues during eating.

. As the infection extends the epithelial cells degenerate and the periodontal fibres are destroyed due to trauma and the action of the proteolytic enzymes liberated in the infected area. This produces a pocket between tooth and alveolus where bacteria and food debris lodge and the toxic discharge from this infected nidus stimulates osteoclasts to absorb the underlying alveolar bone. The pocket extends slowly, pari passu with the absorption of bone, until eventually the tooth becomes loose and is extruded. Long before this occurs, however, the patient suffers from a generalized absorption of toxic matter and, as the disease progresses, showers of organisms escape into the blood stream when food is taken and the soft gum traumatized.

The dangers of such a condition during pregnancy are obvious. It is of the utmost importance that the pathological pockets be eliminated as soon as possible. Removal of teeth will be effective, but the extraction is likely to result in a gross transient bacteriaemia due to the release of organisms into the blood stream. Extractions, therefore, should be limited to two or three at a time and should be accompanied by adequate doses of penicillin.

It is well recognized that the common cause of infective endocarditis is the liberation of streptococcus viridans into the blood stream. As this organism is a common inhabitant of the mouth it is obvious that multiple extractions are a real danger. If a puerperal infection with this organism occurs in a woman who is the subject of an old rheumatic endocarditis the focus in the birth canal may be responsible for a fatal infection of the heart valve (Gibberd, I944).

Treatment. The modern treatment of pyorrhoea aims at removing the pockets by resecting the gum from the tooth as far as the depth of the pocket so that the condition of the mouth is brought back to normal with the difference thae the teeth now protrude more. The patient is then instructed to massage the gums vigorously buk delicately until the surface becomes firmlyø keratinized once again. If this daily routine ofmassage by wood points and soft brush is con $\overrightarrow{\vec{c}}$ tinued the gums remain firm and hard indefinitely? and extension of the damage need not be feared든

The old adage that a woman loses a tooth fote every child she bears may have some point when it is appreciated that during pregnancy the gum? epithelium becomes soft and swollen and is easilyes bruised. Organisms gain entry into the deepero tissues and the cycle of parodontal disease alreadydescribed occurs. At the same time quantities of soft, swollen epithelial cells from the Malphigiano layer of the gum instead of flattening and becoming keratinized in the normal way are exfoliated ano mix with the mouth flora. A sticky, viscid solu $\infty$ tion results which together with a soft, starchy diet clings around the interstitial surfaces of the teeth and in the pits and fissures of the molare crowns. It is generally admitted that herein lieso the predisposing cause of dental caries.

If the ulcers at the edge of the gum can bes healed and the keratinized layer of the gum restored and kept firm and hard, then the conditionmay be said to be cured. Organisms will not $\$$ e् thrust into the deeper tissues and no pus will found round the gum margin. If the pockets not eradicated and the interdental gum margins remain inaccessible then the gum resection has not been sufficiently radical and the condition wilk relapse.

Acute ulcerative gingivitis or Vincent's stomatitis is not so common in civilian life, being found more often in communities such as Army camps. The cases met in private practice are usually slight and present no great problem. The patient complainso that the gums bleed and are painful, whilse clinical examination shows that the tips of the interdental papillae are covered with a smalt adherent slough. The affected parts bleed freely? on the slightest interference and in severe cases the ulceration is deep with an offensive and typicab odour. Cervical adenitis and pyrexia may be present and even septicaemia and death are noE. unknown. In untreated cases which subsideN leading to a chronic marginal gingivitis, there is generally a recurrence every few months and the attachment of the teeth undergoes progressives destruction.

The treatment of the acute symptoms shoul\& aim at preventing the constant tearing of the ulcers at the gum margins. Protection is most easily acquired by painting the ulcers with chromig acid followed by hydrogen peroxide. Free oxygero is liberated and a dark solution of chromic salf 
covers the ulcers with an effect similar to that of tanning. The use of the tooth brush is prohibited and, when necessary, the patient is put on a semifluid diet. If the ulceration persists packing of the pockets with a paste of zinc oxide and oil of cloves is recommended, and the packs should cover the whole gum margin like a plaster casing. In this way a protection against injury is obtained and the natural defences of the body are enabled to heal the ulcers. After the packing is removed (usually from four to five days) the acute symptoms will be found to have subsided. It is now essential to institute after-treatment, for failure to eradicate the pockets and harden the gums will almost certainly lead to recurrence of the disease (Fish, 1944).

\section{Dental Caries}

Dental caries is a bacteriological decomposition of the calcified dental tissues which is affected only by local conditions such as food stagnation and fermentation. It has been demonstrated that the calcium content of formed dentine cannot be altered either by the administration of vitamin $D$ or by calcium starvation, and it is even more unlikely that the enamel can be altered (Fish, 1932). The enamel of the fully calcified tooth is, as far as is known, non-vital, and it is therefore unable to resist the action of acidogenic bacteria which occur in recurrent lodgements of carbohydrate food debris.

Marshall Day (1939, 1944) has described the occurrence of almost perfect teeth coincident with osteomalacia which shows that deposition and resorption of bones is not connected with any such process in the teeth. He also reported that a group of children with severe clinical rickets, confirmed radiologically, had almost perfect teeth with a very low incidence of caries and little gross hypoplasia. The diet of the children was deficient in minerals and vitamin $\mathrm{D}$, and there was good reason to believe that the maternal diet was also deficient in calcifying properties during pregnancy and lactation. In Australia the incidence of dental caries is very high notwithstanding the fact that rickets is virtually unknown. It is evident that diets deficient in calcium, phosphorus and the fat soluble vitamins and rich in 'anticalcifying' cereals do not lead to a greater susceptibility to caries. The conclusion drawn is that the 'detergent diet ' theory of Sim Wallace which postulates that the physical nature and cleansing action of the diet is of more importance than other factors in the prevention of caries is borne out. Sepsis and exanthemata in childhood seem to be more potent factors in the causation of hypoplasia and caries than vitamin $\mathrm{D}$ and mineral deficiencies.

If caries is allowed to progress the dental pulp eventually becomes involved usually with con- siderable pain. This pain is due to an acute infection of the pulp resulting from the exposure of the soft connective tissues. Death of the pulp is the usual sequela, followed by an acute periapical abscess and swelling of the adjacent soft tissues. Eventually the condition becomes chronic. The death of a pulp is a common occurrence and may occur without excessive pain. The majority of pulpless teeth are only discovered by the use of radiographs when a radiolucent area is seen surrounding the apex of the infected tooth. Radiographs of teeth are always of value and are especially indicated where the physician suspects an infected nidus.

It will be seen that whereas pyorrhoea may give rise to constantly recurring bacterial showers in the blood stream, the presence of a pulpless tooth will subject the patient to the end products of bacteria which are ensconced in the root itself or, at most, in an apical granuloma. The danger of a subacute endocarditis arising through the effects of a pulpless tooth does not therefore arise. As a result of recent research work (Ross, 1944), it is now appreciated that the pulp of a tooth can be removed and the root canal filled leaving the periapical tissues normal and healthy. The routine condemnation of pulpless teeth is therefore to be deplored.

The incidence of and susceptibility to caries does not vary between pregnant and non-pregnant women when oral hygiene is a constant factor. In some cases an alteration in the personal habits of the pregnant woman, both nutritional and hygienic, may change the composition of the saliva and the media for the oral flora so that the immediate external environment of the enamel may play the major role in the occurrence of any increase in dental caries.

Treatment. Treatment of the teeth during pregnancy by removal of caries and the insertion of fillings is never contra-indicated. It is important to obtain as healthy a mouth as possible before parturition for, in addition to the reasons already mentioned, it is usually the case that for some time after parturition the mother will have less opportunity for visiting her dental practitioner. With the aid of local anaesthesia, either infiltrated or intra-osseus, treatment as extensive as at other times may be given, but it is advisable during the later stages of pregnancy to make each visit shorter than the normal.

\section{Psychological Approach}

Whilst it may be said that a pregnant woman has an alteration in her mental outlook with a consequent possible neglect of oral hygiene, there is also an opportunity presented to the dental surgeon of instructing a patient who is psychologically pre- 
pared to receive advice on the care of her children's teeth.

It is still common to find parents who consider the deciduous dentition to be of little importance and fail to realize that if neglected there will be serious adverse affects on the permanent dentition. It should be stressed that neglect and early loss of deciduous teeth usually results in poor alignment of the permanent successors. At the same time decayed teeth in a child lead quickly to infection of the tooth pulp and periapical tissues with a resulting general physical upset. Loss of appetite and weight, cervical adenitis and general malaise are the common sequelae in a child with a grossly neglected mouth.

Many parents are ignorant of the serious results that may accrue from the habit of thumb or finger sucking. Indeed many mothers have been known to encourage it in order to keep the baby quiet. Once the habit is formed it is sometimes extremely difficult to break, and attention should be given to the question of prevention at the outset. The continued insertion of the thumb for long periods when the jawbone is soft and not fully calcified may result in protrusion of the premaxilla, retrusion of the mandible or, according to the position of the fingers in the mouth, even an open bite. The number of children that are seen with protruding teeth even allowing for the percentage which are due to other causes is a striking testimony to the result of this unfortunate habit.

There is a definite need for the dental surgeon acting in co-operation with the physician to lay the foundations for the dental well-being of future generations. The subject of the first permanent molar which erupts at about the sixth year is one that might well be emphasized. Erupting as it does posteriorly to the deciduous molars this tooth is often mistaken for one of the deciduous series and is thus neglected as regards cleaning and the observation of early caries. Its early loss through caries will upset the normal articulation and alignment of the teeth which, in turn, will be responsible for the formation of 'pocketing' of the gums and the sequelae already outlined.

It is quite common for the lower permanent incisors to erupt before the deciduous teeth are shed. They are then usually posterior to their predecessors and the arrangement will cause some anxiety to the parent until it is explained that normal growth of the new teeth will carry ther into their correct alignment provided that the arches of the jaws are normal.

The knowledge of the time of calcification of the teeth is of importance in estimating the value of the earliest pre-natal care and treatment of the pregnant woman. All the deciduous teeth have commenced calcification before birth and, indee the tooth buds have been given off from the primitive dental lamina at the ninth week of intra uterine life. All the deciduous incisors have completed calcifying at about 18 months of age and absorption of the roots commences at aboufy four years. At birth the important first permanent molar has one cusp calcified and its eruption at about the sixth year of life is followed by its como plete calcification at ten years. In general the calcification of the crown of a tooth may be re garded as being complete one year previous to this date of its eruption.

It will be appreciated that the dental care of $\mathrm{q}$ woman during her pregnancy consists of certain definite factors which together contribute to hep well-being momentarily and for the future. The modern concept of the problem is to concentrate more fully on the soft tissues surrounding the teet for, in comparison, the care and treatment of the teeth themselves differ very slightly from ofho routine attention required by all patients. Witt further education and the increasing facilitieg available to all sections of the population, a greates consciousness is being shown of the importance of dental treatment and advice. The association of loss of teeth with the advent of child bearing iş as has been indicated above, a non-sequitur where the ordinary effects of dental caries are considered. If the care of the pregnant woman's mouth is focused on the parodontal tissues and it can be. certain that she will play her part in the maintenance of her oral hygiene, then it is possible to avoid the subsequent misery of an edentulous mouth.

In the preparation of this paper considerable assistance was given by Mr. Stewart Ross, to whom the author wishes to offer his thanks and to acknowledge the many suggestions and corrections. in the manuscript made by him.

\section{BIBLIOGRAPHY}

BLUM, T. (1931), f. Amer. dent. Ass., 18, 393.

DAY, C. D. MARSHALL (1939), Brit. med. $\mathfrak{f}$., i, 919.

DAY, C. D. MARSHALL (1944), Ibid, 5, 115.

FISH, E. WILFRED (1932), 'Experimental Investigation of Enamel, Dentine and Dental Pulp.' London, John Ball \& Danielsson.

FISH, E. WILFRED, and MACLEAN, I. H. (1936), Brit. dent. F., 61, 336.

FISH, E. WILFRED (1944), 'Parodontal Disease.' London, GIBBERD, G. F. (r944), 'A Short Textbook of Midwifery MILLER, S. C. (1946), 'Oral Diagnosis and Treatment.' Londoen
H. K. Lewis.

OKELL, G. C., and ELLIOTT, S. D, (1935), Lancet, ii, 869 ROSS, W. STEWART (1944), Proc. Roy. Soc. Med., 8, 46 r. ROUND, H., KIRKPATRICK, H. J. R., and HAILS, C. G (1936), Ibid., 29, 1552 .

ZISKIN, D. E. (1937), $\mathscr{F}$. dent. Res., 16, 367.

ZISKIN, BLACKBERT and STOUT (1933), Surg. Gynee Obstet., 6, 719 . 